\title{
Iris Recognition Using Wavelet Transform and Neural Networks
}

\author{
Divya Pirale ${ }^{1}$, Manisha Nirgude ${ }^{2}$, Dr. Sachin Gengje ${ }^{3}$ \\ ${ }^{1}$ PG Scholar, Computer Science and Engineering, WIT College, Solapur University, Solapur, India \\ ${ }^{2}$ Assistant Professor, Information Technology, WIT College, Solapur University, Solapur, India \\ ${ }^{3}$ Professor, Electronics Engineering, WIT College, Solapur University, Solapur, India
}

\begin{abstract}
Now a day's biometrics gaining popularity because of its higher security and precise authentication process. It is the best medium for recognizing the acquired data with the traditional one. Out of various biometrics such as face, ear, voice, palm, fingerprint, signature, hand geometry, iris recognition has been acknowledged as one of the most accurate biometric modalities because of its high recognition rate. The advantage of working with iris recognition is the iris pattern of an individual is same throughout lifetime. So iris pattern never change irrespective of its surrounding conditions. Hence we present a method of recognizing an iris using wavelet transform and Neural Networks. The paper presents the ANN models and gives respective results and comparisons.
\end{abstract}

Keywords: Neural network, Iris recognition and wavelet transform

\section{Introduction}

Biometrics plays an important role in public as well as in private security domain. It adopts a traditional method of identification and verification. Using various physiological and behavioral characteristics, one can identify each individual and differ it from one another. One of the most popular approaches is iris recognition, because each iris unique structure having a complex pattern and iris is a combination of various characteristics like corona, crypts, filaments, freckles, pits, furrows, striations, rings etc [2]. In addition to this the left and the right iris pattern is different for each and every individuals and it is stable throughout the life, hence can be served as a passport or a password that one need not remember but is always present[3]. It is impossible to alter the iris images, because it could lead to blindness. Hence indeed iris is the most safe and reliable biometric pattern that is used in biometric identification system. The example of an iris pattern is shown in Figure 1.

\subsection{Why the iris? [3]}

Iris comprised of many important characteristics as follows:

- Higher Accuracy- Highest accuracy and no wrong matches

- Uniqueness- No two iris patterns are same, even the identical twins iris patterns are differ from each other.

- Stable- Unique pattern of an iris image formed from 10th month of an age; hence it's stable throughout one's lifetime.

- High performance Content- The amount of information that can be stored in a single iris is much greater than any other biometrics.

- Real Time- Allows high speed processing and the individual just need to look into a camera for a few seconds.

\section{Volume 5 Issue 5, May 2016}

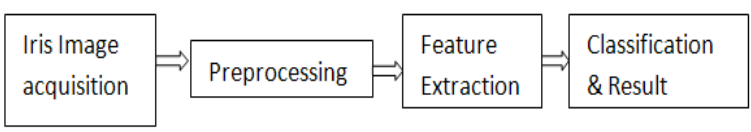

Figure 2: Iris Recognition System

\section{Related Work}

Many researchers worked on iris recognition system, like John Daugman [1] proposed a NIR (near-infrared) wavelength to reveal even darker pigmented iris feature. Here each isolated iris pattern is demodulated to extract its phase information using quadratures 2D-Gabor wavelets. Whereas wavelets which gives the combinational complexity of phase information across different persons over the iris which enables real time decision about personal identity with extremely high confidence. The author tries to give robust representation for pattern recognition in variant to change in 


\section{International Journal of Science and Research (IJSR) \\ ISSN (Online): 2319-7064}

Index Copernicus Value (2013): 6.14 | Impact Factor (2015): 6.391

the size, position and orientation of the pattern.

Leila Fallah Araghi, Hamed Shahhosseini, Farbod Setoudeh [2] proposed the covariance matrix of discrete wavelet transform for edge detection and Probabilistic neural networks and LVQ, or Learning Vector Quantization as a classifier. This approach used to classify noisy image. LVQ is the supervised counterpart of vector quantization system. It is related to self organizing map (SOM). The network has a first competitive layer and a second linear layer.

Usham Dias, Vinita Frietas, Sandeep P.S. and Amanda Fernandes [3] presents personal identification of an iris consist of iris localization, normalization, image enhancement and pattern recognition using neural network. In the addition with this, author successfully shown persons left and right eye is unique. Using Canny Edge detection method edge of an iris is detected. Given network classify sample images of an iris pattern and were trained using error back propagation algorithm with adaptive learning rate (traingda).The experimental results show that the training is sensitive to initial weights and overtraining gives the bad results. Gradient descent with adaptive learning rate backpropogation (traingda) updates weight and bias values according to gradient descent with adaptive learning rate. It sets the network trainFcn property.

R. P. Ramkumar, Dr. S. Arumugam [4] identifies human characteristics by extracting textural feature of a human eye. Pupil localization is done by using negative function and neighbor's method. Histogram equalization technique is used for enhancing the normalized iris image. Hamming distance and cumulative sum based change analysis employed by the author to extract the feature.

Mrunal M Khedkar and S.A Ladakhe [5] proposed an approach where it takes, iris images of 10 different people as an input. After localizing region of interest for extracting the feature the experiment is more focused on image statistics, texture and 2D SVD transform domain. Hence extracted features given as an input to neural network based Pattern Recognition (PR) system. Hence MLP, RBF and SVM are investigated by an author for suitability with PR systems. The experimental results clearly show the performance of MLP (Multi Layer perception) based PR system gives the most consistent results though it retrain with multiple random initialization.

Gajendra Singh Chandel, Ankesh Bhargava [7] proposed an image tool for calculating the length of the iris from left to right and top to bottom. CASIA is used as a database for this approach. Feed forward back propagation algorithm is used a as a classifier for this approach. A vector of predictor variable Values $(\mathrm{x} 1, \mathrm{x} 2)$ are presented at the input layer with number of two neurons. The weighted sum is fed into a transfer function which outputs a value. Bayesian Regulation Training (trainBR) algorithm is used to learn neural network. This function updates the weight and sometime adjusts a weight and bias the value according to Levenberg-Marquardt optimization.

Omaima N. Ahmad AL-Allaf, Abdelfatah Aref Tamimi, and
Shahlla A. AbdAlKader [8] in this proposed approach classification is based on five different ANN (Artificial neural network) models. The training part consists of first determining the architecture of given ANN model then initializing ANN model weights and bias unit further initializing the output target vector for each input vector, so author implemented the experiments on 150 samples. Then the input vector applied to compute output of each layer to find original output vector. Implementation and evaluation done by considering MSE for 4 layers ANN with 5 Hidden Nodes, MSE for 7 layers ANN with 5 Hidden Nodes, effect of Number of Hidden Layers, Effect of Number of Hidden layer Unit, Testing ANN models with Testing Samples. Comparison result shows trainlm is the most suitable algorithms for this proposed work. Trainlm can train any network as long as its weight, net input and transfer function have derivative function. It updates its weight and bias values according to Levenberg-Marguardt optimization.

\section{The Proposed Methodology}

\subsection{Database}

The standard UBIRIS database is used for proposed iris recognition system. Each image of the database is having $200 \times 150$ pixels.

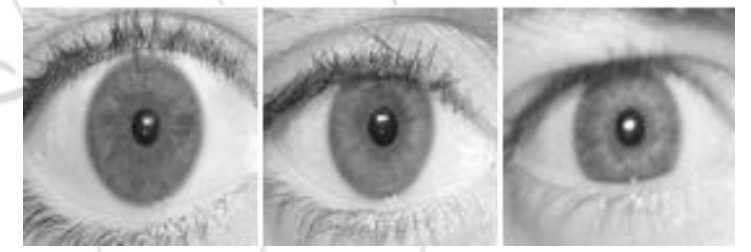

Figure 3: Sample Images of Database

\subsection{Steps in iris Recognition System}

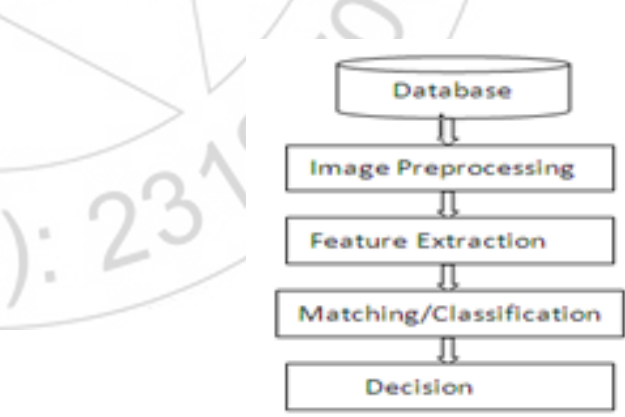

Figure 4: Steps involved in iris recognition system [6]

The steps involved in figure 4 . in iris recognition system are given as follows:

\subsubsection{Image Acquisition}

Iris recognition works by first obtaining an iris image of a person and later stored in database. The database contains all the grayscale images. The total 500 images stored in database.

\subsubsection{Image Pre-processing}

The aim of applying pre-processing to an iris image is to improve image data that enhances some image feature required for further processing. It consists of iris localization 


\section{International Journal of Science and Research (IJSR) \\ ISSN (Online): 2319-7064}

Index Copernicus Value (2013): 6.14 | Impact Factor (2015): 6.391

and pupil circle detection as follows:

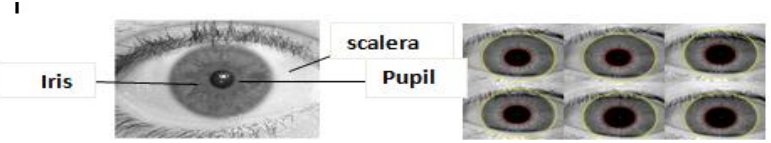

Figure 5: Isolation of an Iris

The iris region is approximated by two circles, as shown in Figure. 5. In order to evaluate an iris localization step, first we separate out an iris from a complete eye image as shown in Figure 6.

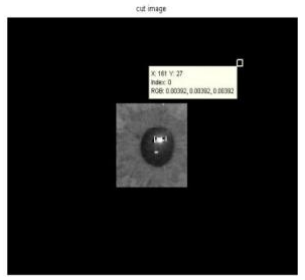

Figure 6: Cut Image

Edges are associated with the boundaries of objects in an iris area. It is mainly used to find the edges in an image. For this approach the canny edge detection method is used [3].we have calculated the center coordinates

The pupil circle detection is a part of iris image preprocessing. An iris image of a database is first converted into a binary image. This can be fulfilled by applying a suitable threshold.

Figure 7 shows the center of pupil and the vertical and horizontal boundary is calculated. Suppose the coordinates for horizontal boundary is $(\mathrm{x} 1, \mathrm{y} 1)$ and $(\mathrm{x} 1, \mathrm{y} 2)$ and for vertical boundary it is $(\mathrm{x} 3, \mathrm{y} 3)$ and $(\mathrm{x} 4, \mathrm{y} 3)$. The following formula (1) is used to find the centre coordinates (Xp, Up) of the pupil [13].

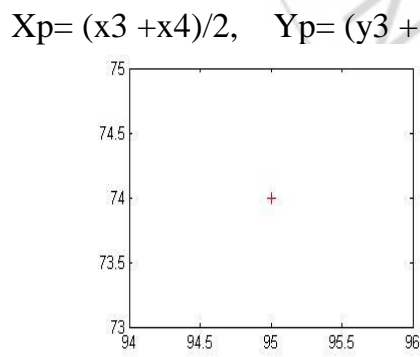

(1)

Figure 7: Center of Pupil

\subsubsection{Iris Segmentation}

Segmentation is the process of portioning the acquired iris image into number of its constituent's parts so that individual regions are indentified perfectly.

\subsubsection{Normalization}

Once the region is successfully segmented from an eye image then the next step is normalizing an iris image. Normalization is an unwrapping process of segmented iris regions that is the transformation process of iris texture from the Cartesian to polar coordinates. Figure 8 is the normalized iris before enhancement and Figure 9 is the enhanced normalized image.
Figure 8: Normalized Iris before enhancement

Figure 9: Normalized Iris after enhancement

\subsubsection{Feature Extraction}

Iris has a unique value and extracting those values is nothing but a feature extraction [2]. Main goal of feature extraction is to extract the features of the given iris and then iris template is generated. Wavelet transform is basically used for extracting the features. For the image processing wavelet transform is used because it analyses an image data and provides precise information about the image. We can use orthogonal wavelets for discrete wavelet transform (DWT) and non orthogonal for continues wavelet transform. As DWT compress an image into suitable format it is any wavelet transform for which wavelets are discretely sampled. Various methods involved in wavelet transform from wavelet families. For example Daubechies, Biorthogonal, Coifletsn, Symlets, Morlet, Harr etc. The proposed approach uses Harr wavelet transform. Features are stored by using Harr wavelet transform. The decomposition is applied to get the features in a more precise format. Harr transform gives the best performance in terms of computation time, very simple to use, and it is one of the efficient method of image compression.

It is an implementation of wavelet transform using a discrete set of wavelets and translation followed by some rules. It is used for numerical and functional analysis. It has a sequence of rescaled square shaped functions.

After applying Harr wavelet transform the decomposed iris image shown in Figure 10 and 11.

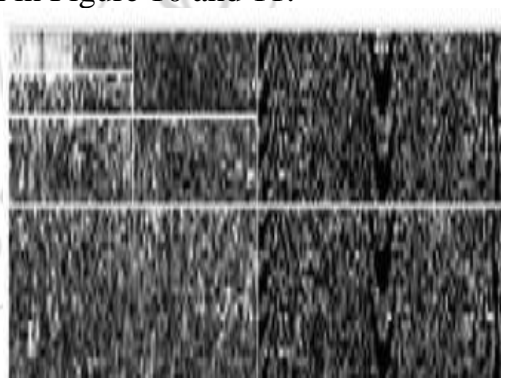

Figure 10: Two Level Decomposition of an iris image

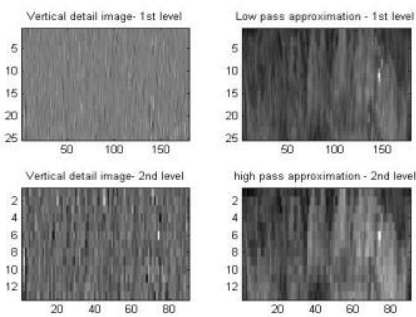

Figure 11: Two level detailed decomposition

\section{Neural Network as a classifier}

The Artificial neural network is used for the classification purpose. It involves training and testing on extracted template of an iris images. Neural network has the ability to 


\section{International Journal of Science and Research (IJSR) \\ ISSN (Online): 2319-7064}

Index Copernicus Value (2013): 6.14 | Impact Factor (2015): 6.391

derive complicated data and also it can be automatically learn how to perform tasks depends on the given training inputs. Below in the results sections we have experimented different ANN algorithm on various number of classes. After the preprocessing and feature extraction, the templates are stored and fed to a neural network for the classification purpose. By implementing different algorithms using ANN, on different number of classes, the results are generated.

\subsection{Feed forward back propagation Neural Networks (FFBPNN)}

A typical feed forward neural network is a type of network architecture where the connections are "fed forward" that is do not form a cycles. This network architecture generally used when we input something at the input layer, it travels from input to hidden layer and again from hidden to output layer. Feed forward with back propagation involves 2 steps 1) Feed Forward the values and 2) propagate back to previous or earlier layer. Back Propagation algorithm, is used to train neural network and to calculate the gradient of the error of the network with respect to its modifiable weights.

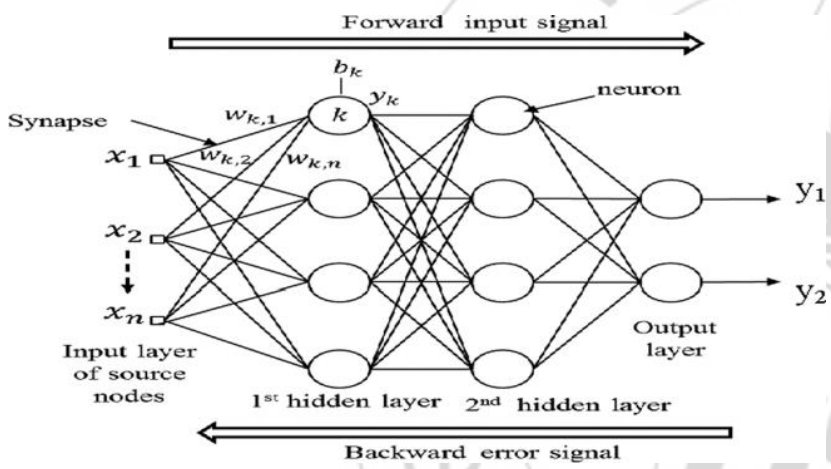

Figure 12: Architecture of FFBPNN [14] The architecture of FFBPNN is as shown in figure 12.

\section{Experimental Results and analysis}

Two different ANN models constructed separately, each with different training function. To evaluate the performance of the constructed models, MSE and the performance accuracy was taken into consideration. In the addition, we also shows the effects of various parameters like number of iterations, performance based on number of classes, epoch etc. as shown below in the tables. The UBIRIS database we are considering, having initially 100 classes with 5 samples of each i.e. $(100 \times 5)=500$. I.e. total 500 inputs. The results analysis divides into two parts i.e. training and testing. For example if we are considering 100 classes $(100 * 5=500)$ then for training parts 300 images were taken and for testing purpose remaining 200 images. The MSE value should kept as low as possible given in formula (5) and accuracy is calculated separately for training and testing as given in formula(6) [5].

$$
\mathrm{MSE}=\frac{\sum_{j=0}^{P} \sum_{i=0}^{N}\left(d_{i j}-y_{i j}\right)^{2}}{N_{\mathrm{P}} P}
$$

Accuracy $=($ No. correct classification $/$ total No. of testing images) $* 100$

\subsection{Levenberg-Marquardt Algorithm}

Feed forward back propagation neural network is used as a neural network using Levenberg-Marquardt (trainLm) as a training function. The best suitable result for the respective network model is as shown below in table 1. It is observed that it is one of the fastest training algorithms for networks of moderate size. It has a memory reduction feature for use when the training set is given. The MSE columns show the performance of an algorithm on given set of inputs. The transfer function used for trainLM is tansig.

Table 1: Result with Levenberg-Marquardt

The various algorithms comes under FFBPNN, among them we have implemented Levenberg-Marquardt Algorithm (trainLM) and Gradient descent algorithm (trainGD).

\subsection{A Two layer feed forward Network}

It is a neural network for pattern recognition. It consists of sigmoid hidden and softmax output neuron. It allows you to select data, create and then train a network and evaluate its performance using cross entropy. The Scaled Conjugate gradient algorithm (trainscg) is used as a training function. It is based on conjugate directions. The analysis of this training function depends upon following measures 1) the best validation performance gained after training the samples and that should be nearer to zero 2) Number of Epoch 3) Gradient measure. The architecture of two layer feed forward network is as shown in Figure 13.

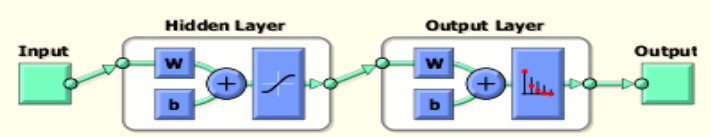

Figure: 13 Architecture of Two layer feed forward network

\begin{tabular}{|l|l|l|l|l|l|l|}
\hline $\begin{array}{c}\text { No of } \\
\text { Classes }\end{array}$ & $\begin{array}{l}\text { No of } \\
\text { Neurons }\end{array}$ & Iterations & MSE & $\begin{array}{l}\text { Training } \\
\text { Accuracy }\end{array}$ & $\begin{array}{l}\text { Testing } \\
\text { Accuracy }\end{array}$ & $\begin{array}{l}\text { Approx } \\
\text { Time }(\mathrm{Hr})\end{array}$ \\
\hline 10 & 13 & 13 & 0.09 & 95.00 & 89.26 & $0: 45$ \\
\hline 25 & 26 & 11 & 0.03 & 94.00 & 90.26 & $1: 00$ \\
\hline 50 & 52 & 17 & 0.07 & 91.26 & 86.00 & $1: 25$ \\
\hline 75 & 66 & 21 & 0.06 & 90.00 & 84.00 & $1: 45$ \\
\hline 100 & 66 & 44 & 0.07 & 90.02 & 80.50 & $2: 04$ \\
\hline
\end{tabular}

\subsection{Gradient Descent Algorithm}

The algorithm is trained for feed forward back propagation neural network. We have divided the classes into number of subgroups. Applying different algorithms on network structure it shows the effectiveness of algorithm and helps us to reach to a proper result. The trainGD algorithm we have used on 100 classes which gives very slow response and it is time consuming as compared to trainLM. We have increased number of neurons to compute better results. But the overall accuracy is decreases. The transfer function used for this approach is tansig. 


\section{International Journal of Science and Research (IJSR) \\ ISSN (Online): 2319-7064}

Index Copernicus Value (2013): 6.14 | Impact Factor (2015): 6.391

Table 2: Results with Gradient descent

\begin{tabular}{|l|l|l|l|l|l|l|}
\hline $\begin{array}{c}\text { No of } \\
\text { Classes }\end{array}$ & $\begin{array}{l}\text { No of } \\
\text { Neurons }\end{array}$ & MSE & $\begin{array}{l}\text { Training } \\
\text { Accuracy }\end{array}$ & $\begin{array}{l}\text { Testing } \\
\text { Accuracy }\end{array}$ & Epochs & $\begin{array}{l}\text { Approx } \\
\text { Time(Hr) }\end{array}$ \\
\hline 10 & 26 & 0.2542 & 91.11 & 89.00 & 125 & $0: 50$ \\
\hline 25 & 39 & 0.1443 & 90.20 & 88.25 & 250 & $1: 25$ \\
\hline 50 & 52 & 0.113 & 89.26 & 84.00 & 450 & $1: 26$ \\
\hline 75 & 65 & 0.1212 & 89.00 & 84.2 & 800 & $1: 45$ \\
\hline 100 & 78 & 0.1413 & 86.25 & 85.21 & 900 & $2: 00$ \\
\hline
\end{tabular}

\subsection{Scaled conjugate Gradient Algorithm}

For this approach, two layer feed forward network is used. It is the only training algorithm that does not require line search [3]. A set of experiments conducted to evaluate the performance of the suggested iris recognition system. The goal of the proposed system is to get the lowest MSE value for every divided class and for the overall 100 classes as well. For trainscg algorithm we have increased number of epochs. Hence it helps to increase the accuracy. The time consumed for training the network is large and it gives results in terms of MSE which is low as compared to traingd.

Table 3: Results with Scaled conjugate gradient

\begin{tabular}{|l|l|l|l|l|l|l|}
\hline $\begin{array}{l}\text { No of } \\
\text { Classes }\end{array}$ & $\begin{array}{l}\text { No of } \\
\text { Neurons }\end{array}$ & MSE & $\begin{array}{l}\text { Training } \\
\text { Accuracy }\end{array}$ & $\begin{array}{l}\text { Testing } \\
\text { Accuracy }\end{array}$ & Epoch & $\begin{array}{l}\text { Approx } \\
\text { Time(Hr) }\end{array}$ \\
\hline 10 & 26 & 0.01 & 97.26 & 90.88 & 1000 & $1: 15$ \\
\hline 25 & 39 & 0.06 & 94.24 & 90.22 & 1000 & $1: 38$ \\
\hline 50 & 52 & 0.07 & 92.00 & 89.45 & 1000 & $1: 45$ \\
\hline 75 & 65 & 0.05 & 91.00 & 88.00 & 1000 & $1: 50$ \\
\hline 100 & 78 & 0.05 & 91.45 & 90.76 & 1000 & $2: 35$ \\
\hline
\end{tabular}

While analyzing these results; it is observed that the more we train the network, the better results we get. Table .4 shows the example of overtraining the results and the approximate time required for training $(100 * 5)$ inputs.

Table 4: Results of Overtraining for 100 Persons

\begin{tabular}{|c|c|c|c|c|}
\hline $\begin{array}{c}\text { No of } \\
\text { Classes }\end{array}$ & $\begin{array}{c}\text { No of } \\
\text { Neurons }\end{array}$ & Iterations & MSE & $\begin{array}{c}\text { Approx } \\
\text { time }(\mathrm{Hr})\end{array}$ \\
\hline 100 & 95 & 111 & 0.06 & 1.10 \\
\hline 100 & 91 & 117 & 0.04 & 1.27 \\
\hline 100 & 97 & 127 & 0.05 & 1.45 \\
\hline 100 & 95 & 110 & 0.04 & 1.50 \\
\hline 100 & 117 & 110 & 0.04 & 1.47 \\
\hline
\end{tabular}

\subsection{Summary results of the above Algorithms}

The above Table shows the best suitable results for this recognition system. The experimental results are as shown in Table. 1, 2 and 3 uses trainLM, trainGD, trainscg algorithms respectively. With this the approximate time required also added, so that it evaluate the time and effectiveness factor of the respective network. MSE value should be as low as possible. Table 3 using (trainscg) i.e. scaled conjugate gradient algorithm gives the highest accuracy for training and testing for the given inputs. The point at which overtraining gives the stable results then accuracy for training and testing moderately increases.

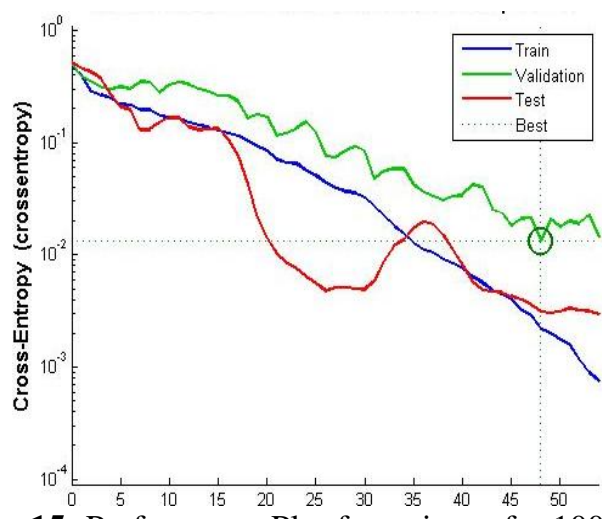

Figure 15: Performance Plot for trainscg for 100 classes

The summary of the above tables are shown below in table 5 . The MSE column shows the performance of the algorithm based on their network structure. Hence it is observed that scaled conjugate gradient algorithm (trainscg) is having the better results as compared to trainLM and trainGD.

Table 5: Summary of the Results

\begin{tabular}{|l|l|l|l|l|}
\hline Algorithm & $\begin{array}{l}\text { Training } \\
\text { Accuracy } \\
(\%)\end{array}$ & $\begin{array}{l}\text { Testing } \\
\text { Accuracy } \\
(\%)\end{array}$ & $\begin{array}{l}\text { Approx } \\
\text { Time } \\
(\text { Hr })\end{array}$ & MSE \\
\hline TrainLM & 90.02 & 80.50 & $2: 04$ & 0.07 \\
\hline TrainGD & 86.25 & 85.21 & $2: 00$ & 0.141 \\
\hline Trainscg & 91.45 & 90.76 & $2: 35$ & 0.05 \\
\hline
\end{tabular}

\section{Conclusion}

A biometric system based on iris using wavelet transform and neural network is presented. The canny method is used for eye detection and further features are extracted using Harr wavelet transform. Extracted features further fed for a neural network for classification purpose. After experimenting with different models of ANN, it was found that the highest accuracy achieved with scaled conjugate gradient algorithm (trainscg) as compared to Levenberg Marquardt algorithm (trainLM) and gradient Decsent algorithm (trainGD). The accuracy we got for training the network for 100 classes is $90.45 \%$ and $90.76 \%$ for testing for trainscg training function. With this it is also observed that training a network repeatedly gives the better result.

\section{References}

[1] How Iris Recognition Works by John Daugman january 2004

[2] Biometric Authentication Systems by Zdene k R iha Vaclav Matyas

[3] A NEURAL NETWORK BASED IRIS RECOGNITION SYSTEM FOR PERSONAL IDENTIFICATION by Usham Dias, Vinita Frietas, Sandeep P.S, and Amanda Fernandes

[4] A NOVEL IRIS RECOGNITION ALGORITHM by R. P. Ramkumar, Dr. S. Arumugam Research Scholar, Mahendra Institute of Technology, Namakkal District Principal, Nandha College of Technology, Erode District. Tamilnadu, India 
[5] Libor Masek, 2003, "Recognition of Human Iris Patterns for Biometric Identification", School of Computer Science and Soft Engineering, The University of Western Australia.

[6] Biometric Techniques Using Neural Networks by Divya Pirale, Manisha Nirgude 2016

[7] Identification of People by Iris Recognition by Gajendra Singh Chandel, Ankesh Bhargava International Journal of Science and Modern Engineering (IJISME) ISSN: 2319-6386, Volume-1, Issue-4, March 2013

[8] www.google.co.in

[9] UBIRIS: A noisy iris image database by Hugo Proenca and Luis A. Alexandre Dep. Informatics, Bolama, 6200-001, Covilha, Portugal

[10] International Journal of Advanced Computer and Mathematical Sciences,HAAR WAVELET TRANSFORM FOR SOLUTION OF IMAGE RETRIEVAL Sangeeta Arora,Yadwinder S. Brar and Sheo Kumar

[11] Rahib H. Abiyev, Koray Altunkaya, 2007, "Neural Network Based Biometric Personal Identification"

[12] Watts, M. and Woodsford, B., "INFO 3312003 Laboratory Workbook", Chapter.6, Lab.6: MLP and Backpropagation.

[13] Personal Iris Recognition Using Neural Network by Rahib H.Abiyev, Koray Altunkaya

[14] www.google.co.in 